\title{
CLASSIFICAÇÃO POR SEQƯENCIAÇÃO: UMA PROPOSTA PARA A DENOMINAÇÃO DOS RAMOS RETARDADOS
}

DALTON DE SOUZA AMORIM

\section{ABSTRACT}

The problem of transposition without distortion of the phylogenetic information of cladograms and trees to a classification has elicited several suggestions from many authors. Both the subordination and sequencing methods have limitations which add up to those intrinsic of the Linnaean system. Conventions were made by Patterson \& Rosen (1977) and Wiley (1979) to solve the problems of fossils in classification, of placing groups of uncertain position, of distinguishing tricotomies from sequencing, and of including the ancestral with recent and other fossil groups in the classification. The impossibility of naming inclusive taxa has been a major problem in the sequenced system.

Herewith a method is proposed to refer to these groups without increasing the number of categories or names. An initial discussion of the relations between sequenced classification and assymetry of the phylogenies is made.

CARACTERIZAÇĀO DOS RAMOS PRECOCE E RETARDADO E A CLASSIFICAÇĀO POR SEQUENCIAÇÃO

O problema da caracterização dos ramos de uma dicotomia será tratado aqui apenas superficialmente, visto que eu o analiso em detalhe em outro trabalho (Amorim, em preparação).

Rosa (1918, 1933), na discussão sobre o formato assimétrico das filogenias - "Rule of Deviation" de Hennig $(1960,1966)$ - mostra que, além da desigualdade em número de espécies atuais que existem em cada um dos ramos, denominados por ele de retardado e precoce, há outras características quase sempre repetidas.

A que mais interessa no momento é a seguinte: embora as espécies atuais do ramo retardado atinjam graus de diferenciação morfológica muito maiores, é o ramo precoce que tem em geral um número significativamente maior de sinapomorfias no nível de separação. Ainda que esta desigualdade no número de caracteres sinapomórficos em cada ramo não ocorra em todas as dicotomias, eła é uma regra muito geral. Este fato pode ser constatado comparando a filogenia de qualquer grupo com sua taxonomia tradicional correspondente: a enorme maioria dos táxons monofiléticos reconhecidos, definidos formalmente e denominados são os ramos precoces (também chamados de laterais ou primitivos). Este fenômeno não parece ser casual, mas resultado de um padrão evolutivo que se repete a cada vicariância. Uma população marginal é isolada do restante dos indivíduos de uma espécie ancestral e, por causa do conjunto de propriedades de cada subpopulação resultante, a tendência é de formar grupos atuais com características diferentes (Amorim, em preparação). Em alguns aspectos, estes fatos já foram anotados por Carson (1959), embora seja possível questionar o grau de influência por ele atribuído à seleção natural (homoseleção); além disso, suas conclusões sobre as "possibilidades evolutivas" do ramo retardado são com certeza errôneas.

A classificação por seqüenciação, portanto, pode ser entendida como a maneira correta de representar a sucessão de eventos assimétricos que ocorrem na evolução dos grupos. 
LIMITAÇOEES DAS CLASSIFICAÇOES E COMO SUPERA-LAS

Quando se obtém uma filogenia para um grupo, é necessário transformá-la, para fins de comunicação, em uma classificação que a reflita de modo integral. Este é um dos pontos mais importantes para a sistemática filogenética (Hennig, 1966, 1975; Nelson, 1972; Rosen, 1974; Wiley, 1979) e também um dos pontos de maior disputa entre as escolas de sistemática (Mayr, 1969, 1974; Sneath \& Sokal, 1973; Ashlock, 1979). As razões da escolha da filogenia como base da classificação já foram discutidas por vários autores nos últimos anos, sendo a principal argumentação a de que todos os tipos de relações entre os organismos podem ser representados e compreendidos por meio do parentesco, o que justamente a torna capaz de servir como um "sistema geral de referência" (Hennig, 1966). Nenhum outro tipo particular de relação tem esta propriedade.

O sistema hierárquico lineano tem limitações que não permitem que a classificação expresse por si só, de modo claro, aspectos mais complexos das relações de parentesco. Diversas propostas foram feitas para superar estas dificuldades, representando de modo inequívoco a filogenia numa classificação (resumidas em Wiley, 1979, 1981, que discute as vantagens de cada uma delas). Aqui serão revistos apenas alguns pontos principais.

Os vários tipos de classificações por subordinação (Hennig, 1969; Griffiths, 1976; Farris, 1976; Lovtrup, 1977; e outros) têm sido menos utilizados nos últimos anos, principalmente por provocarem um incremento muito grande no número de categorias e de nomes supérfluos, tornando-as de memorização e comunicação difíceis (Wiley, 1979). A classificação que utiliza os métodos de seqüenciação e de subordinação conjugados tem sido aceita por muitos autores como a mais apropriada para os fins a que se propõe (Nelson, 1972; Cracraft, 1974; Patterson \& Rosen, 1977; Wiley, 1979). Contudo, o próprio método de seqüenciação tem limitações que, adicionadas àquelas intrínsecas ao sistema lineano, precisam ser superadas.

Esses problemas são discutidos a seguir.

\section{(i) A incorporação de fósseis na classificação, junto com as espécies recentes}

Após alguma discussão (Hennig, 1960; Crowson, 1970; Nelson, 1972; Farris, 1976), Patterson \& Rosen (1977) e depois Wiley (1979) propuseram a classificação conjunta de organismos fósseis e recentes, utilizando algumas convenções. O sistema determina que o táxon fossil ocupe a sua posição filogenética correspondente na lista dos ramos precoces (exceto se sua posição for indeterminada) e que, ao invés de receber uma categoria lineana, o nome do táxon seja acompanhado do nome "Plesion", indicando ser um grupo extinto. Segue um exemplo:

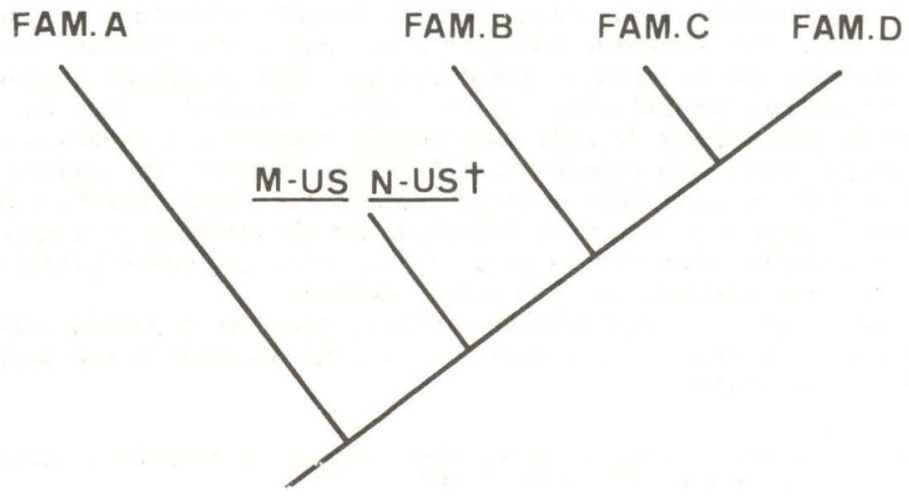




\author{
Ordem X \\ Família A-idae \\ Plesion $M$ - $u$ s $n$ - $u$ s \\ Família B-idae \\ Família C-idae \\ Família D-idae
}

(ii) Incerteza sobre a posição de uma espécie ou um grupo de espécies (atuais ou fósseis) dentro da filogenia de um grupo maior

Dada a necessidade nomenclatural, a dúvida quanto à posição filogenética de um grupo faz com que, na classificação tradicional, ele sempre seja incluído em um táxon maior, com o risco de torná-lo parafilético ou polifilético. Para evitar que haja uma distorção nas relações de parentesco que a classificação filogenética expressa, Patterson \& Rosen (1977) propuseram que os táxons nesta situação não fossem encaixados com os demais na lista da classificação, mas fossem colocados junto ao grupo de menor categoria taxonômica ao qual é possível associá-lo com segurança, acompanhados do nome "incertae sedis".

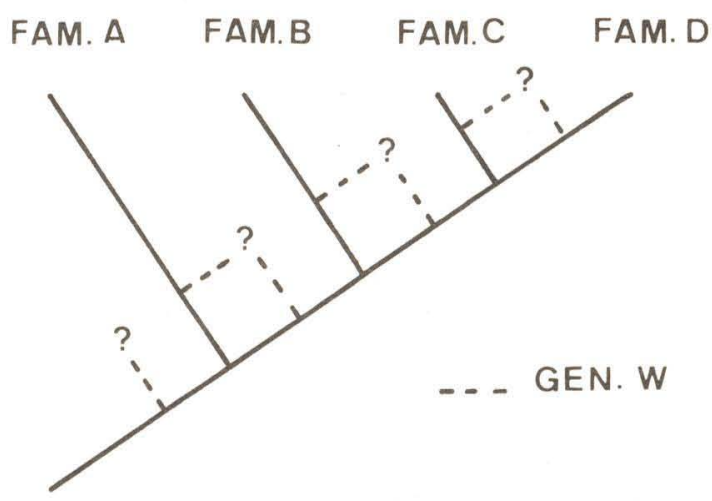

Ordem X

$\mathrm{X}$, "incertae sedis", gên W

Fam. A-idae

Fam. B-idae

Fam. C-idae

Fam. D-idae

(iii) Incerteza no cladograma quanto à proximidade de três ou mais ramos entre si

$\mathrm{Na}$ classificação por seqüenciação três nomes seguidos que correspondam no cladograma a uma tricotomia podem ser tomados como uma simples sequiência, onde o primeiro seria o grupo-irmão dos outros dois, a menos que haja uma indicação explícita (o que não ocorre na subordinação). Wiley (1979) propõe que três ou mais táxons de uma politomia sejam acompanhados de 
"sedis mutabilis", indicando que qualquer deles poderá, com um estudo mais profundo e com a obtenção de novos dados, ser identificado como o grupo-irmão do restante. Isto é, que a seqüência apresentada não corresponde necessariamente à seqüência de divisões e é modificável.
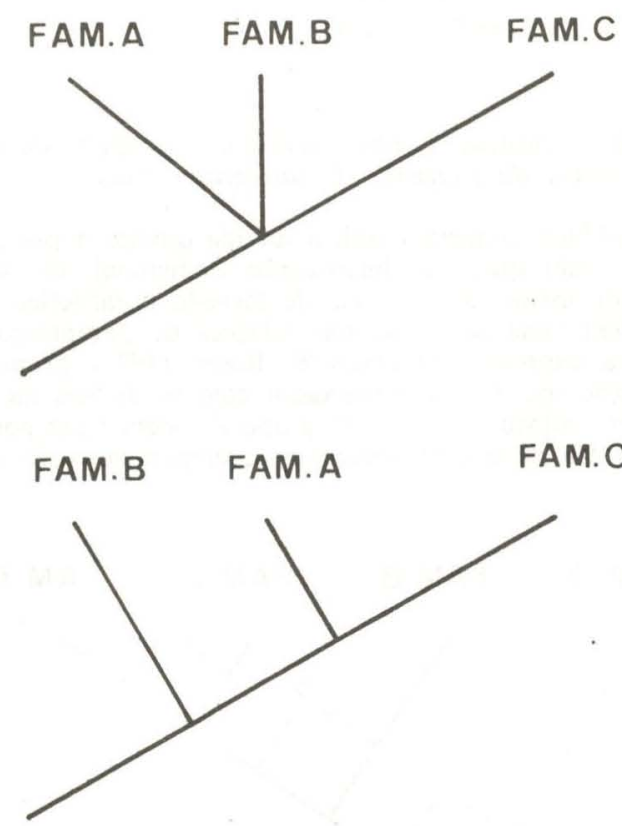

Órdem Y

Ordem $Y$

Fam. A-idae "sedis mutabilis"

Fam. B-idae

Fam. B-idae "sedis mutabilis"

Fam. A-idae

Fam. C-idae "sedis mutabilis"

Fam. C-idae

(iv) Na classificação por seqüenciação um número considerável de grupos monofiléticos fica sem nome

Quando a sequiência dos nomes dos táxons correspondentes aos ramos laterais de uma filogenia é listada no método da seqüienciação, os grupos-irmãos dos sucessivos ramos laterais - ou grupos inclusivos (Platnick, 1977) - ficam sem nome. Às vezes, esses grupos não são de maior relevância. Contudo, numa discussão detalhada da filogenia, analisando cada nível de universalidade, fica muito difícil referir-se a eles e eventualmente alguns táxons podem corresponder a grupos de grande importância evolutiva, taxonômica e/ou biogeográfica: em qualquer caso a ausência de nomes é por muito motivos desinteressante. Este foi o motivo que levou Platnick (1977) a rejeitar a classificação por seqüenciação. Num exemplo, isto pode ser facilmente visualizado: 
GEN.A GEN.B GEN.C GEN.D GEN.E GEN.F GEN.G

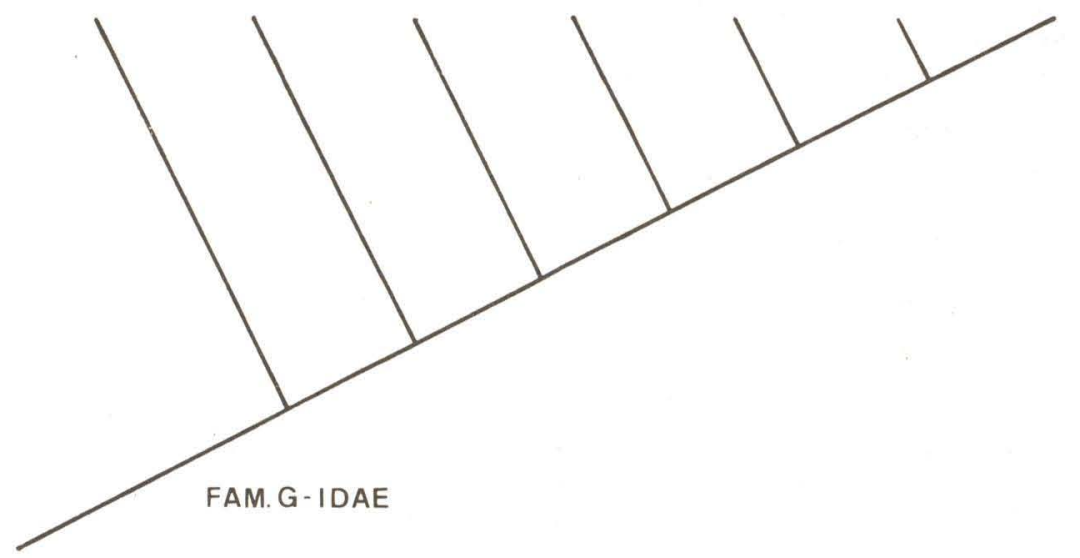

1

Fam. G-idae

gên. A

gên. $B$

gên. C

gên. D

gên $E$

gên. F

gên. G
Fam. G-idae

2

Subfam. A-inae

gên. A

Subfam. G-inae

Supertr. B-idi

gên. B

Supertr. G-idi

Tr. C-ini gên. C

Tr. G-ini

Subtr. D-ina

gên. D

gên. $\mathrm{E}$

gên. F

gên. G

Aqui a classificação por seqüenciação pura (classif. 1) deixa cinco táxons sem denominação própria. Mesmo que seja feita uma classificação mista com a subordinação (classif. 2) e utilizadas as quatro categorias tradicionais, ainda resta um grupo monofilético sem nome. Isto é, abaixo do nível de família, por exemplo, a classificação por seqüenciação de uma filogenia assimétrica com mais de seis terminações deixa pelo menos um grupo sem nome para cada terminação adicional. Se for considerado o número real de dicotomias que existe na maioria quase absoluta de filogenias, fica claro que o número total de táxons nesta situação será enorme. Em Scatopsidae (Diptera), por exemplo, que é uma família pequena, há 13 dicotomias entre o nível de família até o gênero mais distante na filogenia, Quateiella. Sem alterar o conceito de gênero nesta família, que em geral corresponde a grupos mundiais, haveria sete táxons supragenéricos sem nome no ramo principal (e 12 no total); um grupo de importância biogeográfica, como, por exemplo, o grupo mais recente em Scatopsini que ainda não sofreu a divisão da Pangaea, poderia ser denominado "Coboldia + Hawomersleya + Akorhexoza + Quateiella" ou "o grupo-irmão de 
Swammerdamella". A referência ao grupo de qualquer destas duas maneiras é inconveniente, no primeiro caso pela extensão e no segundo por se referir ao grupo utilizando um nome que não faz parte dele (Amorim, 1982).

Uma maneira de superar esta dificuldade seria abreviar esse conjunto de nomes. O táxon seria chamado de "grupo", acrescido do nome do táxon correspondente ao ramo precoce da primeira dicotomia (o primeiro da lista), seguido do sinal "+". No exemplo teórico dado acima ficaria:

$$
\begin{aligned}
& \text { grupo }-\mathrm{B}+\mathrm{C}+\mathrm{D}+\mathrm{E}+\mathrm{F}+\mathrm{G}=\text { grupo }-\mathrm{B}+ \\
& \text { grupo } \cdot \mathrm{C}+\mathrm{D}+\mathrm{E}+\mathrm{F}+\mathrm{G} \quad=\text { grupo } \cdot \mathrm{C}+ \\
& \text { grupo }-\mathrm{D}+\mathrm{E}+\mathrm{F}+\mathrm{G} \quad=\text { grupo } \cdot \mathrm{D}+ \\
& \text { grupo }-\mathrm{E}+\mathrm{F}+\mathrm{G} \quad=\text { grupo } \cdot \mathrm{E}+ \\
& \text { grupo } \cdot \mathrm{F}+\mathrm{G} \quad=\text { grupo } \cdot \mathrm{F}+
\end{aligned}
$$

Embora esse sistema não forneça um nome particular ao grupo, ele preenche plenamente a necessidade de se referir ao táxon de modo inequívoco. $\mathrm{O}$ fato de ser preciso recorrer à filogenia, quando se lida com o nome de um táxon "+" isolado da classificação, para obter a sua localização precisa dentro do grupo, não é uma característica deste artifício. $\mathrm{Na}$ verdade, esta é uma exigência de qualquer classificação biológica. Este trabalho é mesmo facilitado, visto que ele complementa a classificação sem criar nomes adicionais, utilizando um pré-existente: "grupo- $\mathrm{M}+=$ grupo-M mais seu grupo-irmão". Deste modo, o uso deste artifício permite que toda e qualquer filogenia transformada em classificação tenha implícito para cada ramo retardado um nome dado pela própria filogenia, sem que seja necessária uma definição formal. Este nome deixa de ser usado, quando se quiser atribuir ao táxon uma categoria lineana.

\section{(v) Incorporação da espécie ancestral na classificação}

A disputa entre as escolas filogenética e evolucionista sobre a viabilidade de reconhecer o ancestral deixou de ser tão acirrada quanto o foi até o início da década passada, quando os filogeneticistas rejeitavam a metologia de análise gradista e viam com extremo pessimismo esta possibilidade (Hennig, 1966, 1969; Brundin, 1966). A discussão que sucedeu esta, sobre como incorporar a espécie ancestral na classificação, é um indício de que a intransigência inicial deixou de existir, embora poucos (Wiley, 1979, 1981; Schlee, 1981) tenham admitido explicitamente este fato. Muitos autores se mostraram cépticos quanto à possibilidade de classificar espécies ancestrais e recentes conjuntamente (Hennig, 1966; Crowson, 1970; Griffiths, 1974; Patterson \& Rosen, 1977). Contudo Wiley (1979) demonstrou que isso é exequível na subordinação com o uso de artifícios; este autor, contudo, concordou com Nelson (1974) que o método da seqüenciação não comporta a inclusão da espécie ancestral. O uso do "grupo +", como será visto adiante, é uma alternativa que permite solucionar este problema.

A respeito da viabilidade de reconhecer um fóssil como ancestral, alguns pontos merecem ser destacados. Embora Hennig (1966) tenha mostrado que a filogenia de um grupo permite que se acompanhe a evolução de todos os caracteres da holomorfologia desses organismos, a análise de um número muito grande de caracteres não foi feita senão por poucos pesquisadores (Schlee, 1968). Um estudo extenso dos caracteres de um grupo permite traçar o plano-básico de cada grupo monofilético menor dentro da filogenia e, desse modo, detectar ao menos na morfologia externa uma porcentagem significativa das sinapomorfias que surgiram em cada nível de universalidade. $O$ desenvolvimento de técnicas de análise mais refinadas - por exemplo, o "scanning" - amplia continuamente esta percentagem, permitindo o acesso a mais detalhes nos organismos estudados. Por outro lado, as possibilidades de análise 
de fósseis em âmbar, por exemplo, como foi enfatizado por Schlee (1981), é uma das maneiras de comparar estritamente, e às vezes melhor que em alguns exemplares recentes, toda a morfologia e, eventualmente, outros caracteres não morfológicos (veja Schlee, 1981, nota revisionária $7, \mathrm{pg}$. 14). O grande refinamento da análise biogeográfica e a compreensão de como alguns caracteres evoluem (veja adiante), permitem que afirmações sejam feitas nas comparações entre grupos recentes e fósseis com muito mais segurança.

No meu entender, a afirmação de que um fóssil ou um grupo de fósseis correspondam a indivíduos de uma espécie ancestral não é "a priori" uma hipótese indutivista. O fato de esta afirmação não ter nenhuma possibilidade de observação direta não a torna menos testável. Os meios de verificar se um espécime de museu pertence a determinada espécie na natureza não são muito melhores. A tendência em rejeitar essa possibilidade advém justamente de pretender-se uma verificação direta de um fato na natureza, o que, contudo, é sempre crítico no estudo de processos e nas ciências biológicas em geral. Como a observação não é possível, é necessário construir uma hipótese que depois seja testável: a possibilidade que permanece sempre em aberto de descobrir uma autapomorfia do fóssil que o afaste do plano-básico é um meio contínuo de refutar ou corroborar a hipótese com novos caracteres que sejam estudados. De qualquer modo, mesmo que a construção deste tipo de hipóteses for aceita, é necessário ter sempre em mente a relevância desta questão, que é destacado por Nelson (1974, p. 350) com grande clareza:

"Such groups of ancestors seem not to be particularly useful or meaningful taxonomically, and it is only when they can be arbitrarily restricted that the convention of grouping ancestors may be discussed in any semipractical way - provided that there are some known units that may, indeed, be assumed to be ancestral. One may contemplate, for example, the contrast in relevance of "groups" such as the ancestors of the ostrich (the "proostriches"), the "possum (the "pro'possums"), or the perch (the "properches") versus the descendants of the ancestral vertebrate (the Vertebrata), the ancestral gnathostome (the Gnathostomata), or the ancestral teleostome (the Teleostomi)".

Em resumo, o problema dos ancestrais no estudo da evolução é apenas um dentre muitos outros bem mais importantes que ele.

A utilização do artifício proposto no ítem (4) permite que um grupo ancestral seja incluído na classificação de grupos recentes e de grupos do tipo "Plesion". Dentro de uma sequiência um grupo pode ser colocado antes do conjunto de táxons do qual se supõe que ele seja ancestral, com o nome do respectivo "grupo +" seguido, entre parênteses, como sugere Wiley (1979), de "ancest.", e o nome científico do fóssil. Como a hipótese afirma que o fóssil encontrado pertence à população que deu origem aos ramos descendentes, a idade do fóssil data as dicotomias anterior e posterior e sua inclusão é de grande utilidade no incremento do conteúdo da classificação. $\mathrm{Na}$ Figura está representada a filogenia do grupo-Holoplagia + (Seatopsini; Scatopsidae: Diptera) (Amorim, 1982) e abaixo estão as classificações do grupo antes e depois da descoberta de um fóssil $A$-us b-us do grupo-Colobostema +

grupo-Holoplagia +
Holoplagia
Colobostema
Rhexoza
Swammerdamella
grupo-Coboldia +
grupo-Holoplagia +
Holoplagia
grupo-Colobostema + [“ancest." A-us (Triássico)]
Colobostema
Rhexoza
Swammerdamella
grupo-Coboldia +




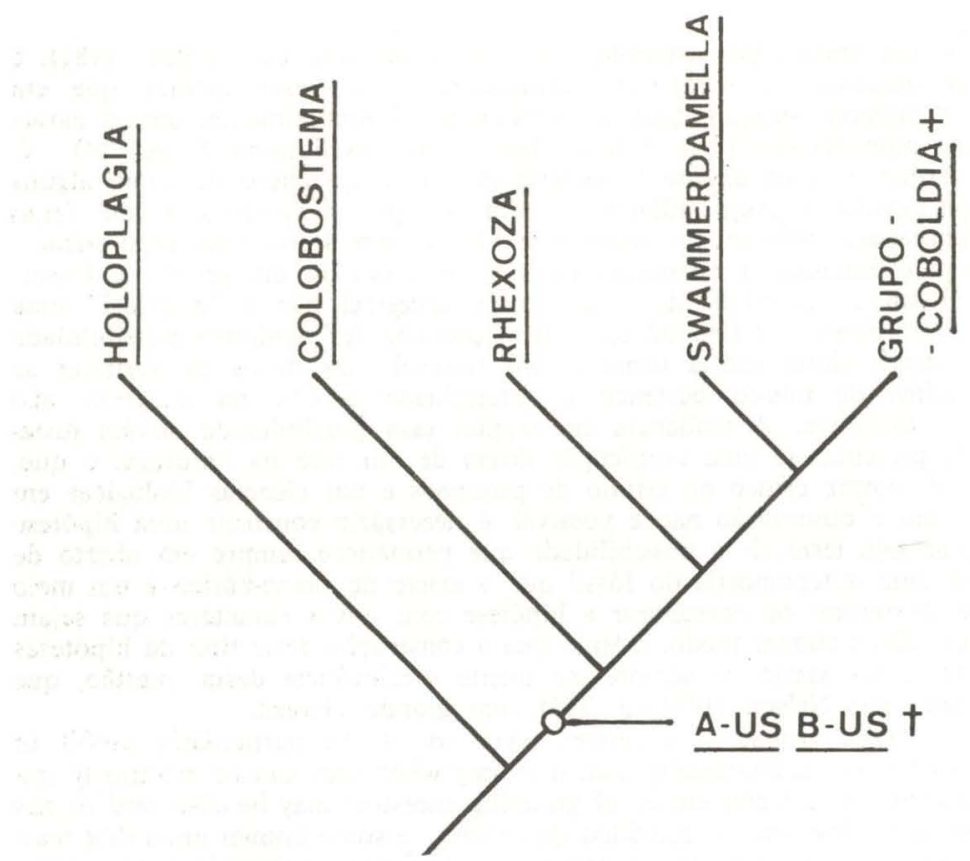

Desse modo, a inclusão de um ancestral não interfere na classificação dos grupos atuais e sua eventual refutação como ancestral deste grupo ou mesmo como ancestral não altera o restante da classificação. Certamente deve haver um limite mínimo de condições de preservação do fóssil e de estudo do grupo para que qualquer afirmação a este respeito possa ser feita. Os animais preservados em âmbar ou aqueles de que é possível, através de uma série muito grande de espécimes do mesmo estrato, reconstituir bastante bem a morfologia, e analisando cuidadosamente os caracteres dos grupos atuais, são os mais recomendados. "Fósseis" que são constituídos de apenas dentes, ossos, pegadas ou quaisquer outras partes do indivíduo total devem ser quase que incondicionalmente colocados em "incertae sedis", mesmo que a estrutura do dente, por exemplo, for idêntica ao do plano-básico. A própria dificuldade de incluir algum grupo "incertae sedis" num ponto interno da classificação por seqüenciação é resolvida pelo uso do "grupo +".

Finalmente, é necessário observar que embora o uso deste artifício permita a inclusão de uma espécie ancestral na classificação, ela não resolve outros pontos muito delicados. A criação para a espécie ancestral de um binômio próprio e independente do seu grupo descendente é, por várias razões, incorreto e inadequado. A sugestão de Griffiths (1974) de abandonar o sistema lineano para a denominação das "time-extended species" poderia ser utilizado nesses casos particulares em que se hipotetiza sobre a ancestralidade de uma espécie, entremeando, portanto, ambos os sistemas com uma complementação recíproca e sem prejuízo a nenhum deles. Para tanto, seria necessário construir novas convenções, o que ainda exige uma discussão mais acurada.

\section{AGRADECIMENTOS}

Este trabalho faz parte da discussão incluída na minha tese de mestrado. A realização de toda a tese foi acompanhada de contribuições preciosas de todos os colegas, amigos e professores que colaboraram comigo e aos quais volto a agradecer. Particularmente, sou profundamente grato ao meu orientador, Dr. Nelson Papavero, que cuidou com um apoio contínuo e encorajador deste período de minha formação. 


\section{REFERENCIAS}

Amorim, D. S., 1982. Sistemática filogenética dos Scatopsidae (Diptera, Oligoneura, Bibionomorpha). Dissertação de Mestrado, Instituto de Biociências, Universidade de São Paulo.

Amorim, D. S. (em preparação). Vicariance and asymmetry of the phylogenies. Ashlock, P. D., 1979. An evolutionary systematist's view of classification. Syst. Zool. 28: 441-450.

Brundin, L., 1966. Transantarctic relationships and their significance, as evidenced by chironomid midges. K. svenska Vet. Akad. Handl. 11: 1-472.

Carson, H. L., 1959. Genetic conditions which promote or retard the formation of species. Cold Spring Harbor Symp. quant. Biol. 24: 87-105.

Cracraft, J., 1974. Phylogenetic models and classification. Syst. Zool. 21: 126-128.

Crowson, R. A., 1970. Classification and biology. Atherton Press, Inc., New York \& Heinemann Educational Books, Ltd., London.

Farris, J. S., 1976. Phylogenetic classification of fossils with Recent groups. Syst. Zool. 25: 271-282.

Griffiths, G. C. D., 1974. Some fundamental problems in biological classification. Ibid. 22: 338-343.

Griffiths, G. C. D., 1976. The future of Linnaean nomenclature. Ibid. 25: 168-173.

Hennig, W., 1960. Die Dipteren-Fauna von Neuseeland als systematisches und tiergeographisches Problem. Beitr. Ent. 10(3/4: 221-329.

Hennig, W., 1966. Phylogenetic systematics, viii $+236 \mathrm{pp}$. University of Illinois Press, Urbana.

Hennig, W., 1969. Die Stammesgeschichte der Insekten, 436 pp. Waldemar Kramer, Frankfurt a. M.

Hennig, W., 1975. Cladistic analysis or cladistic classification? A reply to Ernst Mayr. Syst. Zool. 24: 244-256.

Lovtrup, S., 1977. Phylogeny of Vertebrata. John Wiley \& Sons, Inc., N. York.

Mayr, E., 1969. Principles of systematic Zoology. McGraw-Hill Book Co., N. York.

Mayr, E., 1974. Cladistic analysis or cladistic classification? Z. zool. Syst. Evol.-Forsch. 12: 94-128.

Nelson, G. J., 1972. Phylogenetic relationship and classification. Syst. Zool. 21: $227-231$.

Nelson, G. J., 1974. Classification as an expression of phylogenetic relationships. Ibid. 22: 344-359.

Patterson, C. \& D. E. Rosen, 1977. Review of ichthyodectiform and other Mesozoic teleost fishes and the theory and practice of classifying fossils. Bull. Am. Mus. nat. Hist. 158: 81-172.

Platnick, N. I., 1977. The hypochiloid spiders: A cladistic analysis, with notes on the Atypoidea (Arachnida, Araneae). Am. Mus. Nov. 2627: 1-23.

Rosa, D., 1918. L'Ologenesi. Bemparad, Firenze.

Rosa, D., 1931. L'Ologénèse: Nouvelle théorie de l'évolution et de la distribution géographique des êtres vivants, xii $+368 \mathrm{pp}$. F. Alcan, Paris.

Rosen, D. E., 1974. Cladism or gradism? A reply to Ernst Mayr. Syst. Zool. 23: $446-451$.

Schlee, D., 1968. Vergleichende Merkmalanalyse zur Morphologie und Phylogenie der Corynoneura-Gruppe (Diptera, Chironomidae). Stuttg. Beitr. Naturkde 180: 1-150.

Schlee, D., 1981, in W. Hennig, Insect phylogeny (2nd ed.), xxii + 514 pp. John Wiley, Chichester.

Sneath, P. H. A. \& R. R. Sokal, 1973. Numerical taxonomy. W. H. Freeman, San Francisco.

Wiley, E. O., 1979. An annotated Linnaean hierarchy, with comments on natural taxa and competing systems. Syst. Zool. 28: 308-337.

Wiley, E. O., 1981. Phylogenetics: The theory and practice of phylogenetic systematics. John Wiley and Sons, N. York. 